\title{
Influence of Gender on Therapy and Outcome of Neuroendocrine Tumors of Gastroenteropancreatic Origin: A Single-Center Analysis
}

\author{
Martina T. Mogl ${ }^{\mathrm{a}} \quad$ Eva M. Dobrindt ${ }^{\mathrm{a}} \quad$ Josephine Buschermöhle ${ }^{c}$ Claudia Bures $^{\mathrm{a}}$ \\ Johann Pratschke $^{\mathrm{a}}$ Holger Amthauer $^{\mathrm{b}}$ Christoph Wetz ${ }^{\mathrm{b}}$ Henning Jann ${ }^{\mathrm{c}}$ \\ ${ }^{a}$ Department of Surgery, Campus Charité Mitte, Campus Virchow-Klinikum, Charité - Universitätsmedizin Berlin, \\ corporate member of Freie Universität Berlin, Humboldt-Universität zu Berlin, and Berlin Institute of Health, Berlin,

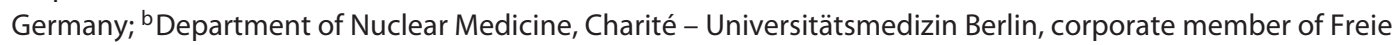 \\ Universität Berlin, Humboldt-Universität zu Berlin, and Berlin Institute of Health, Berlin, Germany; ' Department of \\ Hepatology and Gastroenterology, Campus Virchow-Klinikum and Campus Mitte, Charité - Universitätsmedizin \\ Berlin, corporate member of Freie Universität Berlin, Humboldt-Universität zu Berlin, and Berlin Institute of Health, \\ Berlin, Germany
}

\section{Keywords \\ Gender medicine · Neuroendocrine neoplasm · Liver metastases · Radiotherapy · Chemotherapy}

\begin{abstract}
Introduction: Gender-specific treatment is gaining growing attention in various fields of medicine. In gastrointestinal cancer, influence of sex on outcome has been discussed, while this has not been the case in neuroendocrine tumors. Overall, the incidence of neuroendocrine neoplasms is rising, especially for appendiceal neuroendocrine neoplasms in women. Also, women seem to have a slight advantage in response to therapy, especially for liver metastases. Objectives: This single-center analysis aimed to investigate gender-specific differences in our cohort related to distribution, therapy, and outcome. Methods: Patients from the NET registry as well as the clinic database were evaluated retrospectively concerning overall survival and response to therapy with respect to gender. A subgroup analysis was carried out for patients with low grading and response to chemotherapy, as well as for patients with good and moderate grading receiving peptide receptor radionuclide therapy and for a group of patients with liver surgery. Results: No specific differences could be detected for overall survival or response
\end{abstract}

to therapy between male and female patients. Mean survival was estimated with 242.2 months ( \pm 10.39 SD) altogether and 221.7 months ( \pm 13.02 SD) for male patients and 253.5 months $( \pm 15.24$ SD) for female patients from the NET registry from initial diagnosis. There was no significant difference between female and male patients $(p=0.136)$. For patients receiving chemotherapy, overall survival from initial diagnosis was calculated with 26 months $( \pm 2.59)$ and did not show any significant differences between female and male patients 24.8 months ( \pm 2.81 SD) vs. 27.8 months ( \pm 3.86 $\mathrm{SD}, p=0.87)$. Patients undergoing peptide receptor radionuclide therapy showed a median progression-free survival of 26.9 months ( \pm 2.82 SD), with 16.9 ( \pm 5.595 SD) and 26.9 months $( \pm 3.019$ SD) for male and female patients, respectively $(p=0.2)$. In the group of patients with liver surgery, female patients reached an estimated overall survival of 64.7 months $( \pm 4.16 \mathrm{SD})$, male patients 65.1 months $( \pm 2.79$ $\mathrm{SD}, p=0.562)$. Conclusion: Our cohort did not reveal significant differences in outcome and response to therapy with regards to gender.

(c) 2020 S. Karger AG, Basel 


\section{Introduction}

A remarkable difference exists in epidemiology of neuroendocrine neoplasms (NEN) for male and female patients concerning both incidence and prevalence. Worldwide, the incidence of NEN of gastroenteropancreatic origin (GEP-NEN) has been increasing from 1.09 per 100,000 per year in 1973 to 6.98 per 100,000 per year in 2012 [1]. The incidence rate in Norway, for example, increased from 13.3/100,000 persons per year in 1993 to $21.3 / 100,000$ persons per year in 2010 , as published by Boyar Cetinkaya et al. [2]. The annual growth in incidence rates was $5.1 \%$ for women and $2.1 \%$ for men. In Austria, the incidence for NEN was 2.51 per 100,000 male inhabitants and 2.36 per 100,000 female inhabitants in 2010 [3]. In the US, prevalence was generally higher in female patients and highest in the cohort aged 55-64 years [4]. Also, female patients are more likely to develop stomach, appendiceal, and coecum NEN, while men may develop ileal and rectal NEN [5]. A large nationwide study in Israel could show that female sex was associated with elevated risk for developing appendiceal NEN, while male sex was linked to significantly elevated risk for developing ileal NEN [6]. This could be verified in the UK, where appendiceal NEN showed the greatest absolute increase in incidence between 1971 and 2006 and already were the most frequent GEP-NEN in women in 1990 [7].

Patients with GEP-NEN have various treatment options. Cornerstone of any therapy is surgery with individualized approaches as far as timing and extent are concerned. Especially resection of the primary tumor is known to positively influence outcome [8]. Due to often long-time survival of patients with GEP-NEN, various systemic therapies are important, ranging from somatostatin analogs and mammalian target of rapamycin (mTOR) inhibitors, that is, rapamycin to cytotoxic chemotherapy and peptide receptor radionuclide therapy (PRRT) in metastatic disease [9]. Chemotherapy includes carboplatin-based regimens with etoposide or folate/5-fluorouracil (FOLFOX), while PRRT has been established with Lutetium-177-(DOTA[0]-Phe[1]Tyr[3])octreotide (DOTATOC) as receptor-specific therapy [10].

Patients may undergo repeated surgery and various treatment modalities during the course of disease. This allows for long-term survival, even in metastatic disease [8]. Prognosis is negatively correlated with age $>50$ years, duodenal or pancreatic primary, poor differentiation, and tumor size $>2 \mathrm{~cm}$ at initial diagnosis $[1,11]$.

The influence of gender on therapeutic strategies in NEN has not been of concern in the clinic so far [12]. Gender-specific medicine respects individual reactions of men and women to clinical treatment due to immu- nological, hormonal disparities or unknown cause. This retrospective single-center analysis aimed to evaluate sex-related differences in outcome of GEP-NEN.

\section{Materials and Methods}

Patients from our center were analyzed using the NET registry as well as prospectively collected data from our clinical database. All patients have given written informed consent for participation in the registry, data collection, and analysis in accordance to the Declaration of Helsinki and approved by the local Ethics Committee. 293 patients from our tertiary referral center were available for further analysis from the NET registry, with initial diagnoses from January 1, 1991 until December 31, 2017 and a mean follow-up of 96 months. As mentioned above, there are multiple treatment options for patients with GEP-NEN, especially in metastatic disease, requiring sometimes individual approaches and interdisciplinary well-considered discussion. Therefore, we focused on 3 different treatment options for metastatic GEP-NEN - liver surgery, PRRT, and chemotherapy. One group included patients with low differentiation (G3) undergoing chemotherapy for advanced disease. Therefore, medical records of patients with NEN G3 who presented between $01 / 2013$ and 11/2018 were investigated. One hundred and five patients with histologically confirmed neuroendocrine carcinoma (NEC), neuroendocrine tumor G3, or mixed neuroendocrine non-neuroendocrine neoplasm (MiNEN) G3 who had received chemotherapy as first-line therapy, either before surgery or once they had a recurrence after surgery, were included into this retrospective cohort study. As PRRT is only suitable for better differentiated neoplasms, we analyzed 88 patients with NEN G1/G2. They were treated with Lu-177-DOTATOC PRRT. Patients with follow-up $>12$ months were analyzed for progression-free survival and toxicity. We identified 41 patients, who underwent liver surgery for metastatic NEN between 01/2013 and 11/2018 at our center. Basic demographic variables as well as functionality, location of primary tumor, histological grading, and Ki67 index, as well as outcome were recorded.

Statistical analysis was performed using SPSS version 24 (IBM, Armonk, NY, USA). Overall and progression-free survival time was calculated from the date of diagnosis or intervention to the date of last follow-up or the time of death. Cumulative event rates were calculated using the method of Kaplan-Meier. Univariate analyses were performed using the log-rank test to compare differences between categorical groups. Cox proportional hazards models were developed using relevant clinicopathologic variables to determine the association of each variable with overall survival. $p<0.05$ was considered significant for 2 -tailed probability.

\section{Results}

Patients from the NET registry were evenly distributed between the sexes. 149 of 293 patients (50.8\%) were male, the mean age of all patients at diagnosis was 57.14 years $( \pm 12.75)$, and most patients were diagnosed between 60 and 69 years of age, with no difference between sexes. A majority of patients presented with metastatic disease at time of diagnosis (150 patients, $51.2 \%$ ), while 98 patients (33.4\%) were diagnosed without metastases and 40 patients (13.7\%) with metastasized disease and cancer of 
Table 1. Distribution of primary tumors in female and male patients from the NET registry

\begin{tabular}{lccc}
\hline Primary tumor & $\begin{array}{c}\text { Females (\% of } \\
\text { total females) }\end{array}$ & $\begin{array}{c}\text { Males (\% of } \\
\text { total males) }\end{array}$ & Total (\%) \\
\hline Ileum & $43(29.5)$ & $41(27.5)$ & $84(28.5)$ \\
Jejunum & $1(0.7)$ & $7(4.7)$ & $8(2.7)$ \\
Pancreas & $33(22.6)$ & $38(25.5)$ & $71(24.1)$ \\
Appendix & $8(5.5)$ & $1(0.7)$ & $9(3.1)$ \\
Coecum & $4(2.7)$ & $1(0.7)$ & $5(1.7)$ \\
Colorectal & $8(5.5)$ & $6(4)$ & $14(4.7)$ \\
Stomach & $12(8.2)$ & $11(7.4)$ & $23(7.8)$ \\
Duodenum & $8(5.5)$ & $7(4.7)$ & $15(5.1)$ \\
Lung & $9(6.2)$ & $7(4.7)$ & $16(5.4)$ \\
Cancer of unknown & & & \\
$\quad$ primary & $16(11)$ & $24(16.1)$ & $40(13.6)$ \\
Other & $4(2.7)$ & $6(4)$ & $10(3.4)$ \\
\hline Total & $146(100)$ & $149(100)$ & $295(100)$ \\
\hline
\end{tabular}

Table 2. Distribution of primary tumors (NET G3, NEC, MiNEN G3) in female and male patients receiving chemotherapy

\begin{tabular}{lccc}
\hline & $\begin{array}{c}\text { Females (\% of } \\
\text { total females) }\end{array}$ & $\begin{array}{c}\text { Males (\% of } \\
\text { total males) }\end{array}$ & Total (\%) \\
\hline $\begin{array}{l}\text { Cancer of unknown } \\
\text { primary }\end{array}$ & $14(30.4)$ & $18(30.5)$ & $32(30.5)$ \\
$\begin{array}{l}\text { Esophagus } \\
\text { Stomach }\end{array}$ & 0 & $5(8.5)$ & $5(4.8)$ \\
Pancreas & $2(4.3)$ & $2(3.4)$ & $4(3.8)$ \\
Duodenum & $13(28.3)$ & $14(23.7)$ & $27(25.7)$ \\
Small intestine & $1(2.2)$ & $1(1.7)$ & $2(1.9)$ \\
Colon & 0 & $1(1.7)$ & $1(1.0)$ \\
Rectum & $6(13)$ & $5(8.5)$ & $11(10.5)$ \\
Appendix & $3(6.5)$ & $8(13.6)$ & $11(10.5)$ \\
Bladder & $3(6.5)$ & 0 & $3(2.9)$ \\
Prostate & $2(4.3)$ & $2(3.4)$ & $4(3.8)$ \\
Cervix uteri, ovary & 0 & $3(5.1)$ & $3(2.9)$ \\
Total & $2(4.3)$ & 0 & $2(1.9)$ \\
\hline
\end{tabular}

unknown primary. Only $29.7 \%(n=87)$ of patients presented functionally active disease, mostly with carcinoid syndrome (36 male and 30 female patients). Specific hormone secretion was detected in 14 patients $(2 \times$ insulin, $7 \times$ gastrin, $4 \times$ glucagon, $1 \times$ VIP) .

Localization of primary tumors was evenly distributed between sexes. Most patients (31.4\%) were found to have NEN of small intestine (jejunum, ileum) and pancreas (25\%) followed by colorectal and gastroduodenal manifestation of the primary tumor (Table 1). The majority of patients was diagnosed with good $(\mathrm{G} 1=87$ patients, $29.7 \%$ ) or moderate ( $\mathrm{G} 2=93$ patients, $31.7 \%)$ grading according to the NET registry. No statistically significant difference could be calculated related to sex. While 50 patients of the cohort presented with complete remission at

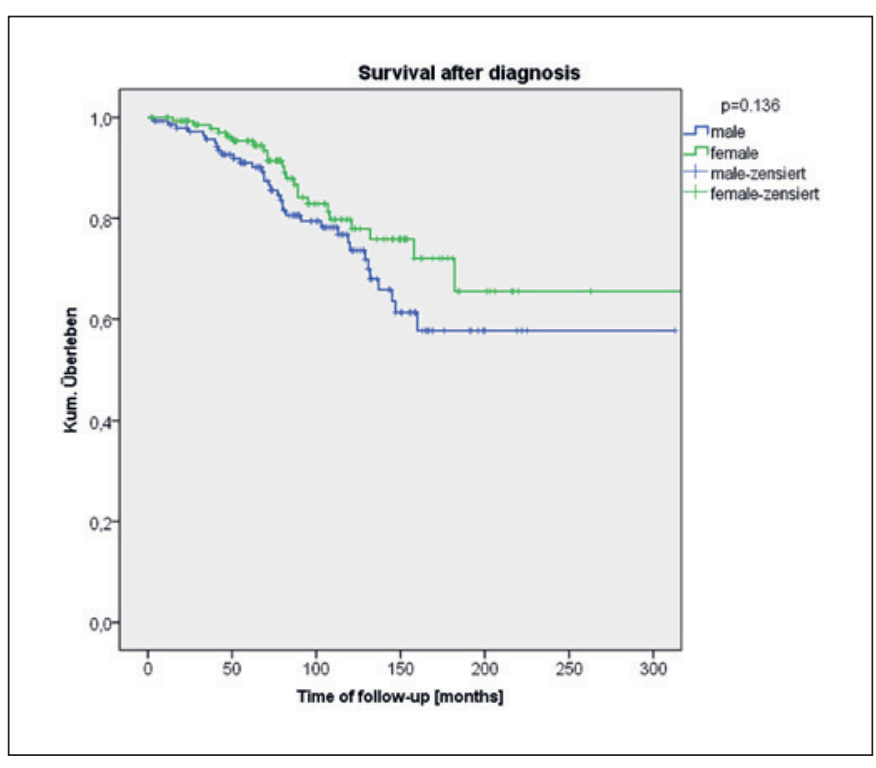

Fig. 1. Kaplan-Meier curve displaying overall survival after diagnosis for female (green) and male (blue) patients with neuroendocrine neoplasias independent of therapeutic strategy.

last visit, 94 patients remained with stable disease and 37 patients were registered with progressive disease (15 patients unknown).

Altogether, 58 patients (19.8\%) had died (35 male, 23 female) until the end of follow-up. Mean survival was estimated with 242.2 months ( $\pm 10.39 \mathrm{SD}$ ) altogether and 221.7 months $( \pm 13.02 \mathrm{SD})$ for male patients and 253.5 months $( \pm 15.24$ SD) for female patients from the NET registry from initial diagnosis There was no significant difference between female and male patients $(p=0.136$; Fig. 1).

Concerning the influence of grading on survival, no significant differences could be calculated between sexes. Ninety-nine patients with good grading (G1) had an expected overall survival of 256.77 months $( \pm 18.74$ SD), with 184.33 months $( \pm 11.88 \mathrm{SD})$ and $252.49( \pm 29.87 \mathrm{SD})$ for male and female patients, respectively $(p=0.567)$. One hundred and twenty-nine patients with moderate grading (G2) demonstrated a mean survival of 202.61 months $( \pm 10.42$ SD), with no difference for male $(151.81 \pm 10.42$ months SD) and female (213.42 \pm 13.19 months SD) patients, respectively $(p=0.288)$. Survival was shortest for 13 patients with low grading (G3), reaching 113.92 months ( \pm 20.86 SD) overall and $99.04 \pm 21.34$ vs. 146.33 \pm 31.65 months SD for male and female patients $(p=$ $0.554)$.

\section{Chemotherapy in G3/NEC}

The cohort of patients with NEC, neuroendocrine tumor G3, or MiNEN G3 who had received chemotherapy as first-line therapy also showed no significant differences in view of gender. Forty-six patients (44\%) of the 105 


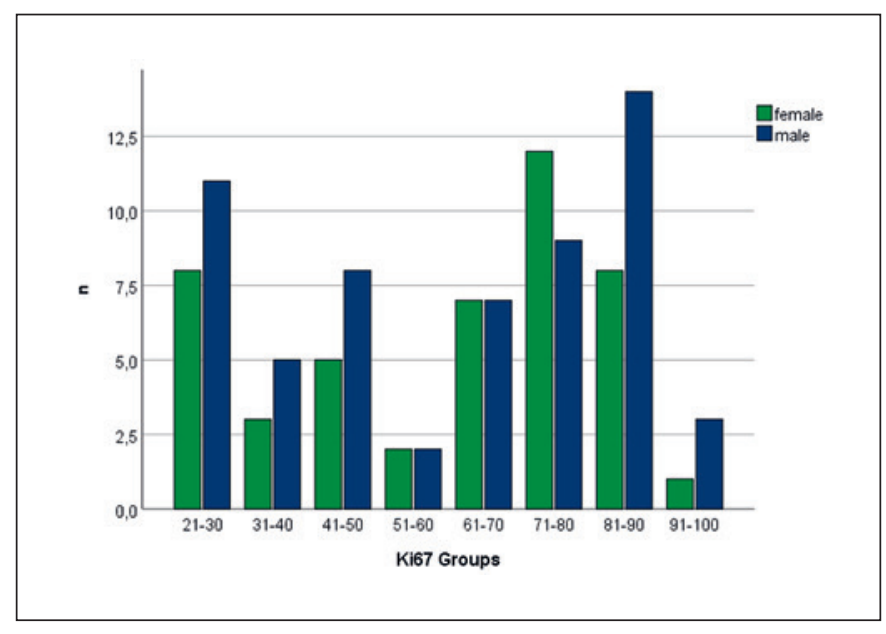

Fig. 2. Illustration of individually highest recorded Ki67 value in percentiles and distribution between female (green) and male (blue) patients for patients receiving chemotherapy.

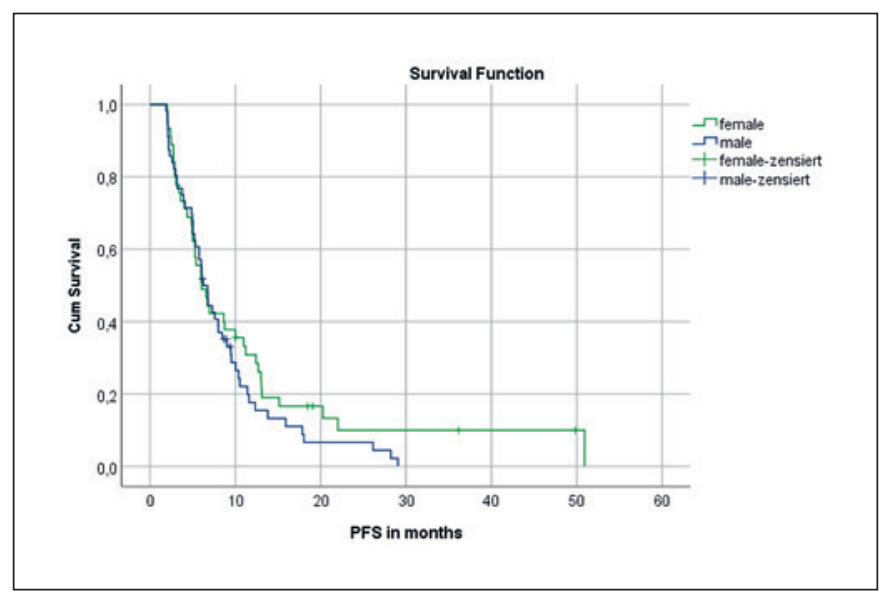

Fig. 3. Kaplan-Meier curve displaying progression-free survival (PFS) of female (green) and male (blue) patients with NEC, NET G3, or MiNEN G3 receiving chemotherapy. Log-rank test.

patients were females. Mean age at diagnosis was 60 years $( \pm 12.37 \mathrm{SD})$ for female and 58.8 years $( \pm 14.14 \mathrm{SD})$ for male patients $(p=0.627)$. The distribution of primary tumors was not significantly different between the sexes $(p=0.564$; Table 2).

When assessing grading of these tumors, the highest recorded Ki67 value was equally distributed between the groups ( $64.1 \pm 22.4$ female vs. $63.6 \pm 24.24$ male, $p=0.927$; Fig. 2). Patients in this cohort with poor grading received various first-line chemotherapeutic regimens with no significant differences in distribution between sexes. FOLFOX was administered to 15 female (14.3\%) and 14 male (13.3\%) patients, cisplatin/etoposide to $16(15.2 \%)$ and 15 (14.3\%) patients, and carboplatin/etoposide to 13 female (12.4\%) and 19 male (18.1\%) patients, respectively. Other regimens were given to 2 female $(1.9 \%)$ and 11 male (10.5\%) patients (FOLFIRI, temozolomide/capecitabin,

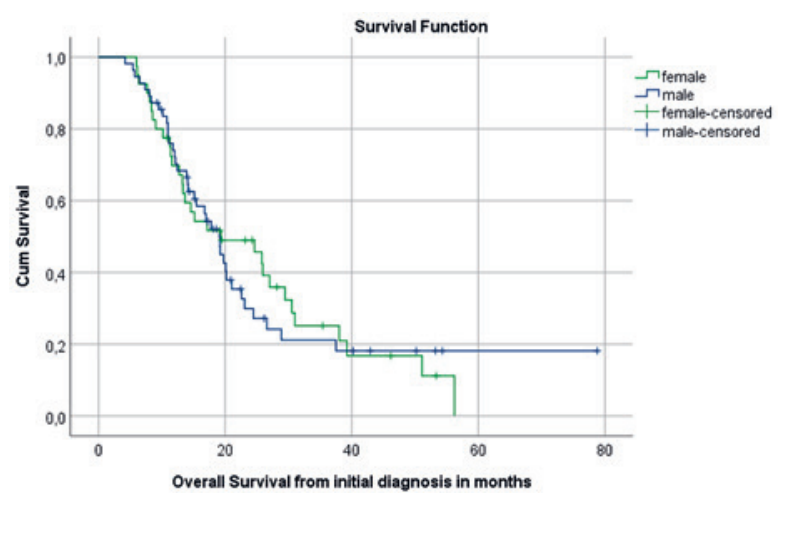

Fig. 4. Kaplan-Meier curve displaying overall survival of female (green) and male (blue) patients with NEC, NET G3, or MiNEN G3 receiving chemotherapy. Log-rank test.

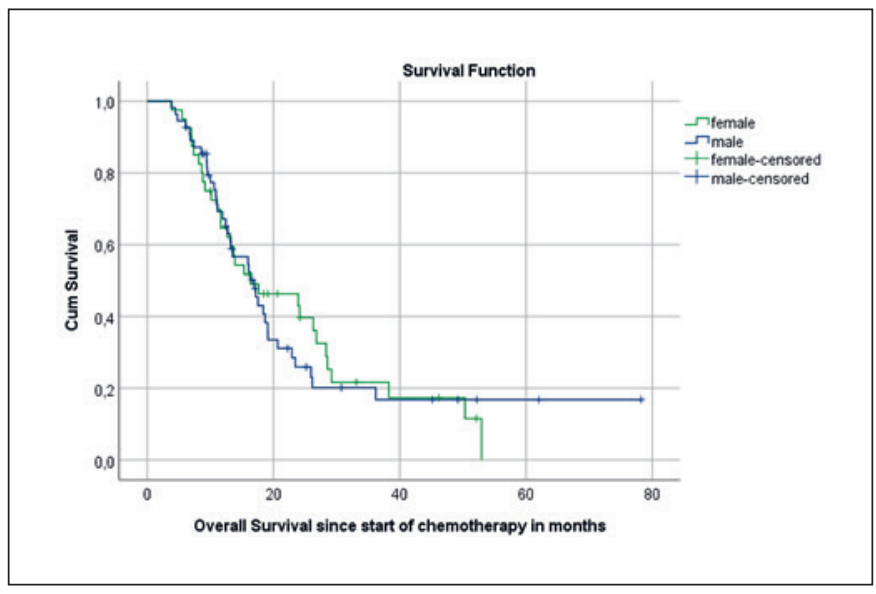

Fig. 5. Kaplan-Meier curve displaying survival since start of chemotherapy of female (green) and male (blue) patients with NEC, NET G3, or MiNEN G3 receiving chemotherapy. Log-rank test.

other). Of this cohort, 19 female (18.1\%) and 18 male (17.1\%) patients had surgery with resection of the primary tumor before (15 patients) or after (22 patients) initiation of first-line chemotherapy, predominantly for gastrointestinal primaries $(n=25,23.8 \%)$. None of these patients was treated with PRRT.

Mean progression-free survival was 10 months in total $( \pm 1.13 \mathrm{SD})$, with no significant difference between gender groups. While women were estimated to reach 11.9 months $( \pm 2.22 \mathrm{SD})$, male patients showed a mean progression-free survival of 8.4 months $( \pm 0.93 \mathrm{SD}, p=0.268$; Fig. 3). Overall survival from initial diagnosis was calculated with 26 months $( \pm 2.59)$ and did not show any significant difference between female and male patients. Female patients reached an overall survival of 24.8 months $( \pm 2.81 \mathrm{SD})$, male patients one of 27.8 months $( \pm 3.86 \mathrm{SD}$, $p=0.87$; Fig. 4). Likewise, survival since start of medical 


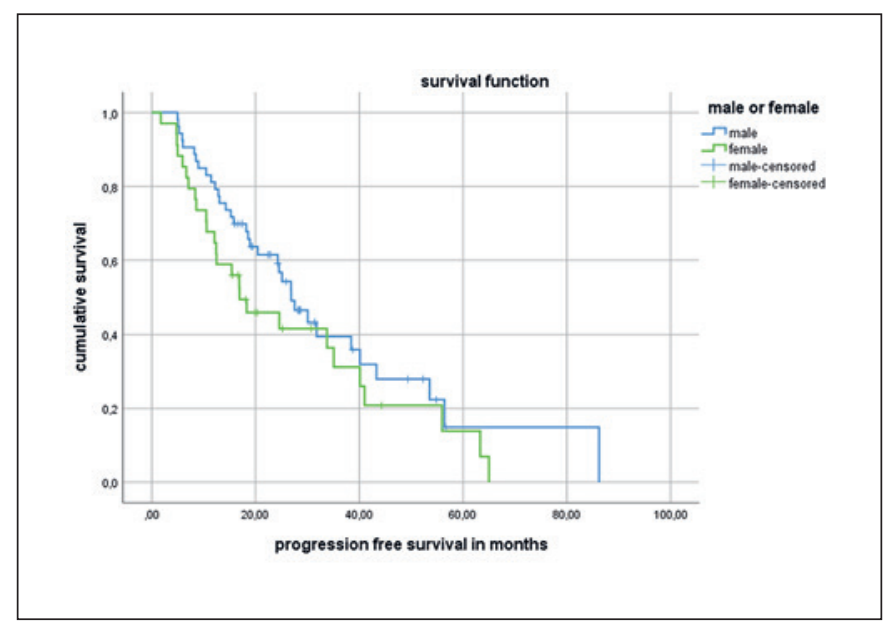

Fig. 6. Kaplan-Meier curve showing progression-free survival after first cycle of Lu-177-DOTATOC-PRRT for male (blue) and female (green) patients with gastroenteropancreatic NEN G1/G2.

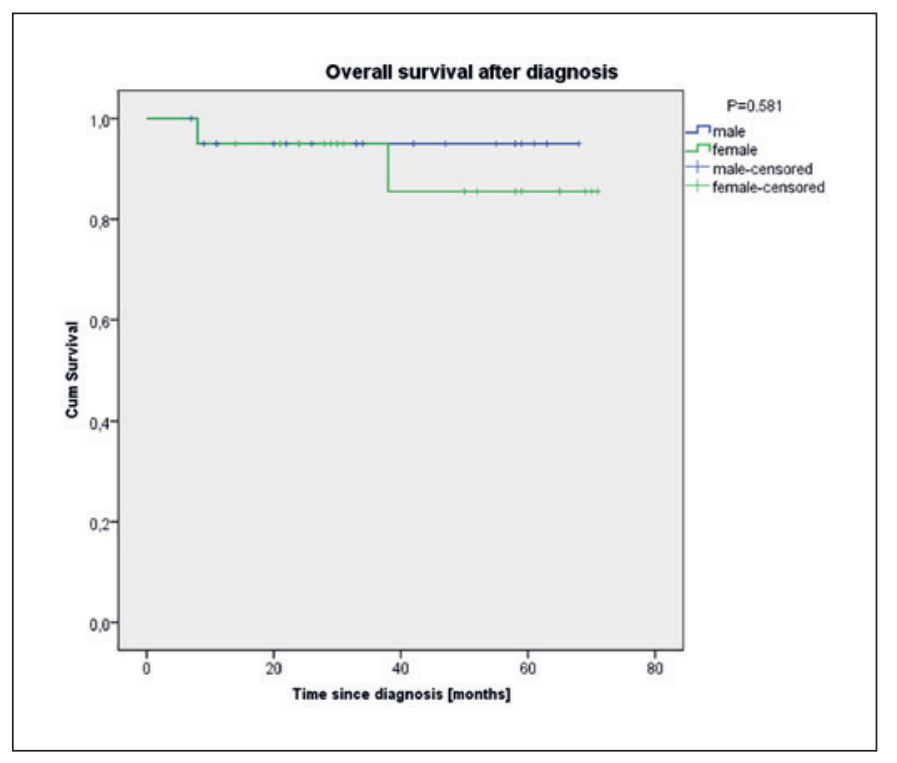

Fig. 7. Kaplan-Meier curve displaying overall survival after diagnosis for male (blue) and female (green) patients undergoing hepatic resection for metastatic neuroendocrine neoplasms.

therapy (with or without operation) did not reveal any differences between the groups (22.94 \pm 2.72 months female vs. $25.78 \pm 3.80$ months male, $p=0.845$; Fig. 5).

\section{PRRT G1/G2}

The group of patients with GEP-NEN G1/G2 receiving PRRT consisted of 54 female (61.4\%) and 34 male (38.6\%) patients. Of these, 44 patients were diagnosed with an ileal primary, 11 with rectal, 19 with pancreas primaries, and 14 patients with cancer of unknown primary. Grading was G1 for 20 patients (22.7\%) and G2 for 68 patients (77.3\%). They underwent a median of 3 cycles of PRRT (range 1-6) with 7.45 GBq Lu-177-DOTATOC per cycle.
Table 3. Distribution of primary tumors in female and male patients with liver surgery for gastroenteropancreatic neuroendocrine neoplasia

\begin{tabular}{llll}
\hline Primary tumor & $\begin{array}{l}\text { Females (\% of } \\
\text { total females) }\end{array}$ & $\begin{array}{l}\text { Males (\% of } \\
\text { total males) }\end{array}$ & Total (\%) \\
\hline Small intestine & $9(45)$ & $7(33.3)$ & $16(39)$ \\
Coecum & $2(10)$ & $2(9.5)$ & $4(9.8)$ \\
Pancreas & $3(15)$ & $5(23.8)$ & $8(19.5)$ \\
Rectum & $1(5)$ & $1(4.8)$ & $2(4.9)$ \\
Lung & $1(5)$ & $1(4.8)$ & $2(4.9)$ \\
Liver & 0 & $2(9.5)$ & $2(4.9)$ \\
Cancer of unknown & & $2(9.5)$ & $3(7.3)$ \\
$\quad$ primary & $1(5)$ & $1(4.8)$ & $4(9.8)$ \\
Other & $3(15)$ & $21(100)$ & $41(100)$ \\
\hline Total & $20(100)$ & & \\
\hline
\end{tabular}

The majority of patients presented with liver (94\%) and lymph node metastases (85\%), followed by bone (36\%), peritoneal (20\%), and lung (5\%) metastases.

Progressive disease was diagnosed in 60 of 88 patients $(66 \%)$ altogether. Twenty-six male $(76.5 \%)$ and 34 female patients $(63 \%)$ were diagnosed with disease progression. Median progression-free survival was 26.9 months $( \pm 2.82$ $\mathrm{SD})$ after the first cycle. Male sex was correlated with progression-free survival of 16.9 months $( \pm 5.595 \mathrm{SD})$ and female sex with 26.9 ( \pm 3.019 SD) months, but without significant difference in univariate Cox regression $(p=0.2$; Fig. 6).

Abdominal pain and nausea were not significantly different between the sexes, with a slight tendency towards male patients (15/34 female vs. $14 / 54$ male patients; $p=$ $0.06)$. Nephrotoxicity occurred in 4 of 88 patients and was not related to sex (male, $n=2$; female, $n=2 ; p=1.0$ ). Myelodysplastic syndrome did not occur in this cohort.

\section{Liver Surgery}

Patients undergoing liver surgery were evenly distributed among the sexes (21 male and 20 female patients). The distribution of primary tumors showed no difference, with most patients showing primary NEN in small intestine $(n=16,39 \%)$, followed by pancreas $(n=8$, $19.5 \%)$ and coecum $(n=4,9.8 \%$; Table 3$)$.

Grading of primary tumors was not different between male and female patients. While 4 men and 6 women had good grading (G1), 10 men and 6 women displayed moderate grading of primary tumors. Tumors with low grading (NEC) were found in 5 men and 5 women undergoing liver resection. Two men had hepatic resection for atypical carcinoid tumor and 1 woman for metastasized MiNEN of the coecum.

Three male and 2 female patients had additional PRRT during the course of disease, 1 male patient was treated 


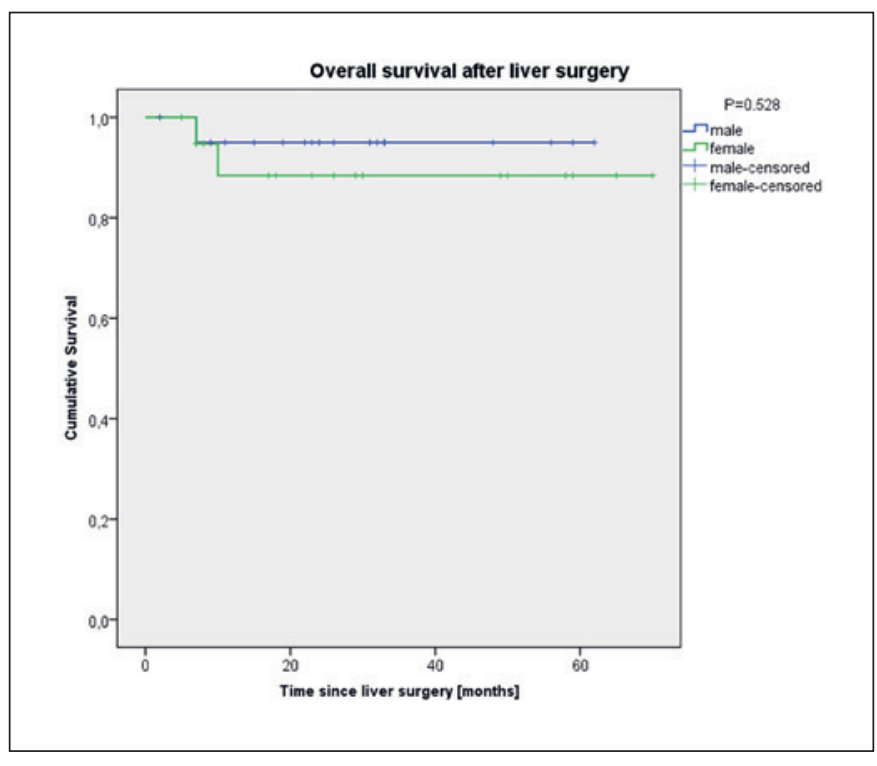

Fig. 8. Kaplan-Meier curve displaying survival after liver surgery for hepatic metastases in male (blue) and female (green) patients with neuroendocrine neoplasms.

with transarterial embolization for liver metastasis, and 1 female patient had high-dose brachytherapy (afterloading) followed by transarterial embolization 2 years later. Selective internal radiotherapy (SIRT) was only used once in a male patient with extended liver metastases 2 years after partial liver resection, and radiofrequency ablation (RFA) in 1 female patient. Interventions were distributed equally between the groups and did not show any significant influence on survival.

Overall survival from initial diagnosis did not show any significant differences between female and male patients $(p=0.58)$. Two male patients had died until the end of follow-up, as well as 3 female patients. While female patients reached an estimated overall survival of 64.7 months $( \pm 4.16 \mathrm{SD})$, male patients were calculated to reach 65.1 months $( \pm 2.79 \mathrm{SD}, p=0.562$; Fig. 7). Likewise, survival since liver operation did not reveal any differences between the groups $(62.9 \pm 4.75$ years female vs. $59.4 \pm 2.56$ years male, $p=0.528$; Fig. 8 ).

\section{Discussion}

This single-center analysis revealed no sex-related differences in outcome for patients with NEN independent of treatment modality. While overall survival reached a mean of 20 years $(242.2 \pm 10.39$ months SD) in the total cohort of patients from the NET registry, not surprisingly, patients with NET G3, NEC, or MiNEN G3 showed lower overall survival of approximately 2.3 years. For this latter group of patients receiving chemotherapy, no sexspecific differences could be calculated, either.
Even though somatostatin analogs are the most often used medical therapy in receptor-positive NEN [13], no data on sex-specific differences in response to treatment, side effects, or outcome exist. The antiproliferative treatment with mTOR inhibitor everolimus is commonly used for treatment of metastasized lung and GEP-NEN, as well as in NEN of unknown origins [14]. Its influence on tumorigenesis and progression of NEN was shown in clinical studies, yet no gender difference could be detected [15]. A trial comparing streptozotocin with dacarbazine/ temozolomide or platin-based chemotherapy could not show gender-specific differences, either. Only age above 55 years and pancreatic NEN phase III were associated with worse survival [16]. Interestingly, a trial on FOLFOX therapy in colon cancer reported influence of muscle mass at the time of diagnosis on discontinuation or dose modification for colon cancer. While association of low muscle mass with discontinuation of chemotherapy was more likely in men, no clear relation to sex could be calculated [17]. No further studies elucidate sex-specific differences concerning response to chemotherapy in NEN. For example, the trial on the effects of octreotide LAR in metastatic neuroendocrine midgut tumors (RPOMID) did not provide data on sex-specific differences in outcome [18], nor did a retrospective analysis of second-line chemotherapy with capecitabine/temozolomide in patients with pancreatic NEN [19]. While this chemotherapeutic regimen has shown good response for patients with GEPNEN G1/G2 in a large single-center series, again no sexspecific effects were reported [20]. Neither did a phase II trial on the effect of temozolomide and thalidomide in patients with metastatic NEN show gender differences [21]. Thus gender-specific aspects with different chemotherapies in GEP-NEN seem to be of lower importance.

PRRT has been shown to achieve good outcome for patients with well- and moderately differentiated GEPNEN. Especially Ki67 index, extent of liver metastases, patients' performance, and neuron-specific enolase levels were independently associated with survival in a retrospective single-center analysis [22]. Gender was not evaluated or did not reach statistical significance in this cohort. A large phase III trial of PRRT with $177-\mathrm{Lu}-\left(\right.$ DOTA $^{0}$, $\mathrm{Tyr}^{3}$ )octreotate (DOTATATE), another widely used agent for treatment of midgut NEN, confirmed the overall benefit of PRRT in comparison to octreotide LAR alone. This was also linked to a positive hazard ratio for both men and women [10]. A small trial on efficacy of PRRT in liver metastases of GEP-NEN could show that male sex and extent of liver metastases were associated with reduced progression-free survival in multivariate analysis [23]. Our analysis of 88 patients receiving PRRT did not reveal any difference in progression-free survival regarding sex. There was a slight tendency of more gastrointestinal side effects of therapy in female patients, 
which did not reach statistical significance. Nephrotoxicity was low and not dependent on gender. No myelodysplastic syndrome occurred in our cohort.

The subgroup analysis of patients undergoing liver resection for metastatic NEN did not show a survival benefit for female compared to male patients. Patients, nevertheless, reached a very good mean overall survival of 5.5 years. Altogether, 4 patients had additional locoregional treatment of the liver including RFA, SIRT, and TAE, which were distributed equally between the groups. However, no biostatistical analysis was performed on these data.

Considering various aspects of therapy for liver metastases of NEN, Karabulut et al. [24] could show that women with liver metastases and multimodal treatment including resection, embolization, and RFA had the best overall survival. Size of the dominant metastasis and extrahepatic disease were additional factors for survival in multivariate analysis. Comparable data were shown for RFA alone, where male sex was associated with worse survival in a single-center prospective trial on RFA for unresectable liver metastases [25]. This could not be concluded from our data, but our sample size was small. A large retrospective study evaluating surgery for liver metastases of pancreatic NEN neither showed an influence of gender on outcome [26]. This is comparable to a large Asian retrospective trial analyzing the impact of liver surgery on outcome in patients with NEN, which could not demonstrate a significant influence of sex on survival [27]. Here, higher age ( $>50$ years), pancreatic primary, primary size $>3 \mathrm{~cm}$, and sum of hepatic metastases $>5 \mathrm{~cm}$ were shown to negatively influence survival in multivariate analysis. Resection of the primary positively influenced survival in multivariate analysis.

NEN are associated with better survival than many other types of malignant tumors. The greatest influence on overall survival are grading, patient age ( $>65$ years), size of the primary tumor, localization, and lymph node status. Grading of neuroendocrine tumors has been established as percentage of Ki67-positive cells in 10 highpower fields [28]. An interesting study by Hallet et al. [29] could show that higher age, male sex, low socioeconomic status, and living in rural areas were associated with worse overall survival. The 5-year survival for patients with G2/ G3-NEN was 64.8\% (95\% CI 63.3-66.2), while for patients with NEC-G3 5-year survival decreased to $8.4 \%$ (95\% CI 7.8-9.1). In this analysis, women displayed significantly better survival [30]. This was confirmed by a large cohort trial in the US with over 35,000 patients where in NEN-G1/G2, male gender had significantly worse survival [5]. An interesting analysis developing a nomogram for prediction of overall survival in pancreatic NEN calculated that female sex and living in a relationship are linked with improved outcome [31].
In our cohort, no influence of gender on survival could be calculated. This was true for the overall analysis of patients from the NET registry irrespective of treatment modality in our institution. Also, patients with low-grade NEN receiving chemotherapy, as well as patients with PRRT for metastatic disease did not reveal any difference in survival related to sex, nor did the subgroup of patients with liver resection.

Limitations of our study lie in its retrospective nature and small sample size, especially regarding outcome after liver surgery. A clear separation of therapeutic effects could not be achieved due to partial overlap of patients between the treatment groups.

\section{Conclusion}

This single-center retrospective analysis of different treatment modalities in patients with neuroendocrine neoplasia has not shown any sex-specific differences. The growing incidence of GEP-NEN concerns both sexes but is pronounced in women, especially in appendiceal NEN. A clear reason for gender disparity in incidence, therapy response, and outcome has not been defined so far. No gender-specific therapies have been developed and systematic studies are missing.

\section{Acknowledgement}

We would like to acknowledge all persons working in direct patient care in the NET center, especially Corinna Schröder, Susanne Steglich, and Corinna Möbius.

\section{Statement of Ethics}

This is a retrospective non-interventional study. All procedures performed were in accordance with the ethical standards of the institutional research committee and with the 1964 Helsinki Declaration and its later amendments or comparable ethical standards. All patients have given written informed consent for participation in registry, data collection, and analysis in accordance with the Declaration of Helsinki and approved by the local Ethics Committee.

\section{Disclosure Statement}

The authors have no conflicts of interest to declare.

\section{Funding Sources}

All authors are employees of the hospital, no further funding was available for this study. 


\section{Author Contributions}

M.T.M., H.J., and C.W. contributed to the study, conception and design, analysis and interpretation of data, and writing of the manuscript. M.T.M., J.B., E.M.D., H.J., C.W., and C.B. participated in the acquisition and analysis of data. J.P. and H.A. contributed to the analysis and interpretation of data, the study conception, and critical review of the manuscript.

\section{References}

1 Dasari A, Shen C, Halperin D, Zhao B, Zhou $\mathrm{S}, \mathrm{Xu} \mathrm{Y}$, et al. Trends in the Incidence, Prevalence, and Survival Outcomes in Patients With Neuroendocrine Tumors in the United States. JAMA Oncol. 2017 Oct;3(10):133542.

2 Boyar Cetinkaya R, Aagnes B, Thiis-Evensen E, Tretli S, Bergestuen DS, Hansen S. Trends in Incidence of Neuroendocrine Neoplasms in Norway: A Report of 16,075 Cases from 1993 through 2010. Neuroendocrinology. 2017;104(1):1-10.

3 Niederle MB, Hackl M, Kaserer K, Niederle B. Gastroenteropancreatic neuroendocrine tumours: the current incidence and staging based on the WHO and European Neuroendocrine Tumour Society classification: an analysis based on prospectively collected parameters. Endocr Relat Cancer. 2010 Oct 17(4):909-18.

4 Broder MS, Cai B, Chang E, Neary MP. Epidemiology of gastrointestinal neuroendocrine tumors in a U.S. commercially insured population. Endocr Pract. 2017 Oct;23(10): 1210-6.

5 Yao JC, Hassan M, Phan A, Dagohoy C, Leary C, Mares JE, et al. One hundred years after "carcinoid": epidemiology of and prognostic factors for neuroendocrine tumors in 35,825 cases in the United States. J Clin Oncol. 2008 Jun;26(18):3063-72.

6 Katz LH, Levi Z, Twig G, Kark JD, Leiba A Derazne E, et al. Risk factors associated with gastroenteropancreatic neuroendocrine tumors in a cohort of 2.3 million Israeli adolescents. Int J Cancer. 2018 Oct;143(8):1876-83.

7 Ellis L, Shale MJ, Coleman MP. Carcinoid tumors of the gastrointestinal tract: trends in incidence in England since 1971. Am J Gastroenterol. 2010 Dec;105(12):2563-9.

8 Shah CP, Mramba LK, Bishnoi R, Unnikrishnan A, Duff JM, Chandana SR. Survival trends of metastatic small intestinal neuroendocrine tumor: a population-based analysis of SEER database. J Gastrointest Oncol. 2019 Oct;10(5):869-77.

9 Strosberg JR. Systemic treatment of gastroenteropancreatic neuroendocrine tumors (GEP-NETS): current approaches and future options. Endocr Pract. 2014 Feb;20(2):16775.

10 Strosberg J, El-Haddad G, Wolin E, Hendifar A, Yao J, Chasen B, et al.; NETTER-1 Trial Investigators. Phase 3 Trial of 177Lu-Dotatate for Midgut Neuroendocrine Tumors. N Engl J Med. 2017 Jan;376(2):125-35.

11 Assi HA, Mukherjee S, Kunz PL, Machiorlatti M, Vesely S, Pareek V, et al. Surgery Versus Surveillance for Well-Differentiated, Nonfunctional Pancreatic Neuroendocrine Tumors: An 11-Year Analysis of the National Cancer Database. Oncologist. 2019;pii:theonc ologist.2019-0466.
12 Strosberg JR, Halfdanarson TR, Bellizzi AM, Chan JA, Dillon JS, Heaney AP, et al. The North American Neuroendocrine Tumor Society Consensus Guidelines for Surveillance and Medical Management of Midgut Neuroendocrine Tumors. Pancreas. 2017 Jul;46(6): 707-14.

13 Rinke A, Müller HH, Schade-Brittinger C, Klose KJ, Barth P, Wied M, et al.; PROMID Study Group. Placebo-controlled, doubleblind, prospective, randomized study on the effect of octreotide LAR in the control of tumor growth in patients with metastatic neuroendocrine midgut tumors: a report from the PROMID Study Group. J Clin Oncol. 2009 Oct;27(28):4656-63.

14 Yao JC, Fazio N, Singh S, Buzzoni R, Carnaghi C, Wolin E, et al.; RAD001 in Advanced Neuroendocrine Tumours, Fourth Trial (RADIANT-4) Study Group. Everolimus for the treatment of advanced, non-functional neuroendocrine tumours of the lung or gastrointestinal tract (RADIANT-4): a randomised, placebo-controlled, phase 3 study. Lancet. 2016 Mar;387(10022):968-77.

15 Lamberti G, Brighi N, Maggio I, Manuzzi L, Peterle C, Ambrosini V, et al. The Role of mTOR in Neuroendocrine Tumors: Future Cornerstone of a Winning Strategy? Int J Mol Sci. 2018 Mar;19(3):E747.

16 Roquin G, Baudin E, Lombard-Bohas C, Cadiot G, Dominguez S, Guimbaud R, et al. Chemotherapy for Well-Differentiated Pancreatic Neuroendocrine Tumours with a Ki-67 Index $\geq 10 \%$ : Is There a More Effective Antitumour Regimen? A Retrospective Multicentre Study of the French Group of Endocrine Tumours (GTE). Neuroendocrinology. 2018;106(1):38-46.

17 Cespedes Feliciano EM, Lee VS, Prado CM, Meyerhardt JA, Alexeeff S, Kroenke CH, et al. Muscle mass at the time of diagnosis of nonmetastatic colon cancer and early discontinuation of chemotherapy, delays, and dose reductions on adjuvant FOLFOX: the C-SCANS study. Cancer. 2017 Dec;123(24):4868-77.

18 Rinke A, Wittenberg M, Schade-Brittinger C, Aminossadati B, Ronicke E, Gress TM, et al.; PROMID Study Group. Placebo-Controlled, Double-Blind, Prospective, Randomized Study on the Effect of Octreotide LAR in the Control of Tumor Growth in Patients with Metastatic Neuroendocrine Midgut Tumors (PROMID): Results of Long-Term Survival. Neuroendocrinology. 2017;104(1):26-32.

19 Saif MW, Kaley K, Brennan M, Garcon MC, Rodriguez G, Rodriguez T. A retrospective study of capecitabine/temozolomide (CAPTEM) regimen in the treatment of metastatic pancreatic neuroendocrine tumors (pNETs) after failing previous therapy. JOP. 2013 Sep;14(5):498-501.
20 Crespo G, Jiménez-Fonseca P, Custodio A, López C, Carmona-Bayonas A, Alonso V, et al. Capecitabine and temozolomide in grade 1/2 neuroendocrine tumors: a Spanish multicenter experience. Future Oncol. 2017 Mar; 13(7):615-24

21 Kulke MH, Stuart K, Enzinger PC, Ryan DP, Clark JW, Muzikansky A, et al. Phase II study of temozolomide and thalidomide in patients with metastatic neuroendocrine tumors. J Clin Oncol. 2006 Jan;24(3):401-6.

22 Ezziddin S, Attassi M, Yong-Hing CJ, Ahmadzadehfar H, Willinek W, Grünwald F, et al. Predictors of long-term outcome in patients with well-differentiated gastroenteropancreatic neuroendocrine tumors after peptide receptor radionuclide therapy with 177Lu-octreotate. J Nucl Med. 2014 Feb; 55(2):183-90

23 Vaughan E, Machta J, Walker M, Toumpanakis C, Caplin M, Navalkissoor S. Retreatment with peptide receptor radionuclide therapy in patients with progressing neuroendocrine tumours: efficacy and prognostic factors for response. Br J Radiol. 2018 Nov;91(1091): 20180041.

24 Karabulut K, Akyildiz HY, Lance C, Aucejo F, McLennan G, Agcaoglu O, et al. Multimodality treatment of neuroendocrine liver metastases. Surgery. 2011 Aug;150(2):316-25.

25 Mazzaglia PJ, Berber E, Milas M, Siperstein AE. Laparoscopic radiofrequency ablation of neuroendocrine liver metastases: a 10-year experience evaluating predictors of survival. Surgery. 2007 Jul;142(1):10-9.

26 He CB, Zhang Y, Cai ZY, Lin XJ. The impact of surgery in metastatic pancreatic neuroendocrine tumors: a competing risk analysis. Endocr Connect. 2019 Mar;8(3):239-51.

27 Du S, Wang Z, Sang X, Lu X, Zheng Y, Xu H, et al. Surgical resection improves the outcome of the patients with neuroendocrine tumor liver metastases: large data from Asia. Medicine (Baltimore). 2015 Jan;94(2):e388.

28 Saeger W, Schnabel PA, Komminoth P. [Grading of neuroendocrine tumors]. Pathologe. 2016 Jul;37(4):304-13.

29 Hallet J, Law CH, Cukier M, Saskin R, Liu N, Singh S. Exploring the rising incidence of neuroendocrine tumors: a population-based analysis of epidemiology, metastatic presentation, and outcomes. Cancer. 2015 Feb; 121(4):589-97.

30 Boyar Cetinkaya R, Aagnes B, Myklebust TÅ, Thiis-Evensen E. Survival in neuroendocrine neoplasms; A report from a large Norwegian population-based study. Int J Cancer. 2018 Mar;142(6):1139-47.

31 Miao DL, Song W, Qian J, Zhu ZG, Wu Q, Lv CG, et al. Development and Validation of a Nomogram for Predicting Overall Survival in Pancreatic NeuroendocrineTumors. Transl Oncol. 2018 Oct;11(5):1097-103. 